\title{
On the Differential Rotation of the Polar Caps of Neutron Stars
}

\author{
A. I. Tsygan ${ }^{*}$, D. P. Barsukov ${ }^{1,2}$, O. A. Goglichidze ${ }^{1}$, and D. A. Shalbykov ${ }^{1}$ \\ ${ }^{\prime}$ Ioffe Physical-Technical Institute, Russian Academy of Sciences, \\ ul. Politekhnicheskaya 26, St. Petersburg, 194021 Russia \\ ${ }^{2}$ St. Petersburg State Polytechnical University, St. Petersburg, 195251 Russia \\ Received March 31, 2014
}

\begin{abstract}
The model of a magnetized rotating neutron star with an electric current in the region of its fluid polar magnetic caps is considered. The presence of an electric current leads to differential rotation of the magnetic caps. The rotation structure is determined by the electric current density distribution over the surface. In the simplest axisymmetric configuration, the current flows in one direction near the polar cap center and in the opposite direction in the outer ring (the total current is zero for the neutron star charge conservation). In this case, two rings with opposite directions of rotation appear on the neutron star surface, with the inner ring always lagging behind the star's main rotation. The differential rotation velocity is directly proportional to the electric current density gradient along the polar cap radius. At a width of the region of change in the electric current from 1 to $10^{2} \mathrm{~cm}$ and a period $\sim 1 \mathrm{~s}$ and a magnetic field $B \sim 10^{12} \mathrm{G}$ typical of radio pulsars, the linear differential rotation velocity is $\sim 10^{-2}-10^{-4} \mathrm{~cm} \mathrm{~s}^{-1}$ (corresponding to a revolution time of $\sim 0.1-10 \mathrm{yr}$ ).
\end{abstract}

DOI: $10.1134 / \mathrm{S} 1063773714090047$

Keywords: pulsars, neutron stars.

\section{INTRODUCTION}

For rotating magnetized neutron stars, existing magnetosphere models (Goldreich and Julian 1969; Beskin 2006; Beskin et al. 2013) predict the existence of an electric current in the region of open magnetic field lines. In particular, in the model of a free charge emission (for example, electrons) (Scharlemann et al. 1978; Arons and Scharlemann 1979) and the inner-gap model (Sturrock 1971; Ruderman and Sutherland 1975), the electric current density is

$$
j=-\frac{\Omega B \cos \chi}{2 \pi},
$$

where $\chi$ is the angle between the angular velocity $\boldsymbol{\Omega}$ and the magnetic field $\mathbf{B}$ on the stellar surface. According to the model of current braking of radio pulsar (Jones 1976; Beskin et al. 1983), an oppositely directed current, such that the total electric current is zero and the system's charge remains constant, flows over the side surface of the pulsar flux tube. In this paper, we will assume that the neutron star surface layer is an electrically conducting fluid. This requires a surface temperature higher than $10^{6} \mathrm{~K}$ (Haensel et al. 2007). Note that the regime of a space charge limited flow is realized precisely at such temperatures (Kantor and Tsygan 2003).

\footnotetext{
${ }^{*}$ E-mail: tsygan@astro.ioffe.ru
}

When passing through a fluid conducting layer, the electric current partially flows across the magnetic field lines. In this case, the Lorentz force causing the differential rotation of the fluid acts on the fluid conducting medium. The goal of this paper is to calculate the velocity of this differential rotation within the magnetohydrodynamic approach.

\section{BASIC EQUATIONS}

We will assume that the upper layer of the polar cap with thickness $d$ is an incompressible conducting fluid with a constant density $\rho$, an electric conductivity $\sigma$, and a kinematic viscosity $\nu$. The polar cap radius is $R=R_{\mathrm{ns}} \sqrt{\Omega R_{\mathrm{ns}} / c}$, where $\Omega=2 \pi / P$ is the angular velocity of neutron star rotation, $R_{\mathrm{ns}} \sim$ $10^{6} \mathrm{~cm}$ is its radius. At a pulsar period of $\sim 1 \mathrm{~s}$, the polar cap radius is $\sim 10^{4} \mathrm{~cm}$. The depth of the fluid layer is $d \sim 3 \times 10^{3} \mathrm{~cm}$ (Haensel et al. 2007). Since both the fluid layer depth and the polar cap radius are much smaller than the neutron star radius, we may assume the layer to be flat and the dipole magnetic field $B$ to be uniform and perpendicular to the surface.

A steady flow of conducting incompressible fluid is described by the magnetohydrodynamic equations (Landau and Lifshitz 1984):

$$
(\mathbf{U} \cdot \nabla) \mathbf{U}=-\frac{1}{\rho} \nabla P
$$




$$
\begin{aligned}
+\nu \Delta \mathbf{U}-\frac{1}{4 \pi \rho}(\mathbf{B} \times \operatorname{curl} \mathbf{B})+\mathbf{g}, \\
\operatorname{curl}(\mathbf{U} \times \mathbf{B})+\eta \Delta \mathbf{B}=0, \\
\operatorname{div} \mathbf{U}=0, \\
\operatorname{div} \mathbf{B}=0,
\end{aligned}
$$

where $\mathbf{U}$ is the fluid flow velocity, $P$ is the pressure, $\mathbf{B}$ is the magnetic field strength, $\eta=c^{2} /(4 \pi \sigma)$ is the magnetic diffusivity, and $\mathbf{g}$ is the free-fall acceleration (assumed to be constant, because the layer is thin compared to the neutron star radius). Below, we will assume that the magnetic field and angular velocity vectors are aligned and directed across the layer. In this case, the current density on the neutron star surface is axisymmetric and, hence, it is convenient to use a cylindrical coordinate system $(r, \phi, z)$ with the $z$ axis directed along the outward normal to the layer. For simplicity, we will consider only axisymmetric flows.

The magnetic field corresponding to the currents in the pulsar flux tube (see (1)) is negligible compared to the neutron star field $B$. It allows one to use the perturbation theory. In the zeroth approximation, the fluid layer is in a constant axial magnetic field $B$ and rotates as a solid body with an angular velocity $\Omega$. In this approximation, no electric current is brought to the layer and the solution of Eqs. (2)-(5) is

$$
\begin{gathered}
\mathbf{U}=\Omega r \mathbf{e}_{\phi}, \quad \mathbf{B}=B \mathbf{e}_{z}, \quad \mathbf{j}=0, \\
P=P(r, z)=\rho\left(-g z+\frac{\Omega^{2} r^{2}}{2}\right),
\end{gathered}
$$

where $\mathbf{g}=-g \mathbf{e}_{z}, g=$ const.

Note that a nonzero electric charge density (Goldreich and Julian 1969) and the related azimuthal electric current density exist in a magnetized neutron star in the laboratory frame of reference. However, it is easy to estimate that this azimuthal electric current density is extremely low, being $\Omega R / c \sim 10^{-6}$ of the density of the current flowing along the pulsar flux tube. Accordingly, both the charge density and the corresponding azimuthal current density may be neglected.

To a first approximation, the electric current is incident perpendicularly to the layer surface $(z=0)$. In this case, we will seek a solution of Eqs. (2)-(5) in the form

$$
\begin{gathered}
\mathbf{U}=\Omega r \mathbf{e}_{\phi}+\mathbf{u}, \quad \mathbf{B}=B \mathbf{e}_{z}+\mathbf{b}, \\
P=P(r, z)+p,
\end{gathered}
$$

where $\mathbf{u}, \mathbf{b}$, and $p$ are quantities of the first order of smallness, which allows system (2)-(5) to be linearized in these quantities. To make the linearized system dimensionless, we use $d$ as a unit of length, $B$ as a unit of magnetic field, $\eta / d$ as a unit of velocity, and $\rho \nu \eta / d^{2}$ as a unit of pressure. Using the same symbols for the dimensionless quantities as those for the dimensional ones, we will obtain

$$
\begin{gathered}
-2 \operatorname{Re} u_{\phi}=-\frac{\partial p}{\partial r}+L_{1}\left(u_{r}\right)+\frac{\partial^{2} u_{r}}{\partial z^{2}} \\
-\operatorname{Ha}^{2}\left(\frac{\partial b_{z}}{\partial r}-\frac{\partial b_{r}}{\partial z}\right) \\
2 \operatorname{Re} u_{r}=L_{1}\left(u_{\phi}\right)+\frac{\partial^{2} u_{\phi}}{\partial z^{2}}+\mathrm{Ha}^{2} \frac{\partial b_{\phi}}{\partial z} \\
\frac{\partial p}{\partial z}=L_{0}\left(u_{z}\right)+\frac{\partial^{2} u_{z}}{\partial z^{2}}, \\
-\frac{\partial u_{r}}{\partial z}=L_{1}\left(b_{r}\right)+\frac{\partial^{2} b_{r}}{\partial z^{2}} \\
-\frac{\partial u_{\phi}}{\partial z}=L_{1}\left(b_{\phi}\right)+\frac{\partial^{2} b_{\phi}}{\partial z^{2}} \\
-\frac{\partial u_{z}}{\partial z}=L_{0}\left(b_{z}\right)+\frac{\partial^{2} b_{z}}{\partial z^{2}} \\
\frac{1}{r} \frac{\partial}{\partial r}\left(r u_{r}\right)+\frac{\partial u_{z}}{\partial z}=0 \\
\frac{1}{r} \frac{\partial}{\partial r}\left(r b_{r}\right)+\frac{\partial b_{z}}{\partial z}=0
\end{gathered}
$$

where

$$
L_{0}=\frac{\partial^{2}}{\partial r^{2}}+\frac{1}{r} \frac{\partial}{\partial r}, \quad L_{1}=\frac{\partial^{2}}{\partial r^{2}}+\frac{1}{r} \frac{\partial}{\partial r}-\frac{1}{r^{2}},
$$

and

$$
\mathrm{Re}=\frac{\Omega d^{2}}{\nu}, \quad \mathrm{Ha}=\frac{B d}{(4 \pi \rho \nu \eta)^{1 / 2}}
$$

are the dimensionless Reynolds and Hartmann numbers, respectively.

The operators $L_{0}$ and $L_{1}$ have the Bessel functions $J_{0}(k r)$ and $J_{1}(k r)$ as eigenfunctions finite at zero and bounded at infinity, $L_{0}\left(J_{0}(k r)\right)=-k^{2} J_{0}(k r)$ and $L_{1}\left(J_{1}(k r)\right)=-k^{2} J_{1}(k r)$.

The solution of system (8)-(15) must be real. Accordingly, we represent $\mathbf{u}, \mathbf{b}$, and $p$ as the Hankel integrals over the variable $r$ with a real $0<k<\infty$ (Bateman and Erdeii 1953). We will represent the functions $u_{z}, b_{z}$, and $p$ as the Hankel integrals of the zeroth kind

$$
\begin{aligned}
& f(r, z)=\int_{0}^{\infty} k f^{0}(k, z) J_{0}(k r) d k, \\
& f^{0}(k, z)=\int_{0}^{\infty} r f(r, z) J_{0}(k r) d r
\end{aligned}
$$


and the functions $u_{r}, u_{\phi}, b_{r}$, and $b_{\phi}$ as the Hankel integrals of the first kind

$$
\begin{aligned}
& f(r, z)=\int_{0}^{\infty} k f^{1}(k, z) J_{1}(k r) d k, \\
& f^{1}(k, z)=\int_{0}^{\infty} r f(r, z) J_{1}(k r) d r .
\end{aligned}
$$

For the Hankel transforms, system (8)-(15) takes the form

$$
\begin{gathered}
-2 \operatorname{Re} u_{\phi}^{1}=k p^{0}+\frac{\partial^{2} u_{r}^{1}}{\partial z^{2}}-k^{2} u_{r}^{1} \\
+\operatorname{Ha}^{2}\left(k b_{z}^{0}+\frac{\partial b_{r}^{1}}{\partial z}\right), \\
2 \operatorname{Re} u_{r}^{1}=\frac{\partial^{2} u_{\phi}^{1}}{\partial z^{2}}-k^{2} u_{\phi}^{1}+\mathrm{Ha}^{2} \frac{\partial b_{\phi}^{1}}{\partial z}, \\
\frac{\partial p^{0}}{\partial z}=\frac{\partial^{2} u_{z}^{0}}{\partial z^{2}}-k^{2} u_{z}^{0}, \\
-\frac{\partial u_{r}^{1}}{\partial z}=\frac{\partial^{2} b_{r}^{1}}{\partial z^{2}}-k^{2} b_{r}^{1}, \\
-\frac{\partial u_{\phi}^{1}}{\partial z}=\frac{\partial^{2} b_{\phi}^{1}}{\partial z^{2}}-k^{2} b_{\phi}^{1}, \\
-\frac{\partial u_{z}^{0}}{\partial z}=\frac{\partial^{2} b_{z}^{0}}{\partial z^{2}}-k^{2} b_{z}^{0}, \\
k u_{r}^{1}+\frac{\partial u_{z}^{0}}{\partial z}=0 \\
k b_{r}^{1}+\frac{\partial b_{z}^{0}}{\partial z}=0 .
\end{gathered}
$$

When deriving system (22)-(29), we used the relations

$$
\frac{1}{r} \frac{\partial}{\partial r}\left(r J_{1}(r)\right)=J_{0}(r), \quad \frac{\partial}{\partial r} J_{0}(r)=-J_{1}(r) .
$$

System (22)-(29) is a system of the tenth order in $z$ (note that only three of the four equations (25)-(27) and (29) are linearly independent). Accordingly, to find the solution of the system, we should specify ten boundary conditions. For the free upper boundary $(z=0)$ stationary along the $z$ axis, we have

$$
\frac{\partial u_{r}^{1}}{\partial z}=0, \quad \frac{\partial u_{\phi}^{1}}{\partial z}=0, \quad u_{z}^{0}=0 .
$$

For the lower solid stationary boundary $(z=-1)$, all velocity components are equal to the velocity at the boundary:

$$
u_{r}^{1}=u_{\phi}^{1}=u_{z}^{0}=0 .
$$

For the magnetic field, the boundary condition is determined by the incident current that fixes the azimuthal magnetic field component at the upper boundary and by the magnetic field continuity at the conductor-dielectric (vacuum) boundary:

$$
b_{\phi}^{1}(k, z=0)=b_{0}(k), \quad \frac{\partial b_{z}^{0}}{\partial z}+k b_{z}^{0}=0,
$$

where $b_{0}(k)$ is a function of the variable $k$ whose form is determined by the electric current density on the surface. Indeed, the electric current density $j_{z}(r, z)$ and the azimuthal magnetic field component $b_{\phi}(r, z)$ are related via Maxwell's equation (we use $c B /(4 \pi d)$ as a unit for the current density)

$$
j_{z}(r, z)=\frac{1}{r} \frac{\partial}{\partial r}\left(r b_{\phi}\right),
$$

which leads to the relation between their Hankel transforms

$$
b_{\phi}^{1}(k, z)=\frac{j_{z}^{0}(k, z)}{k},
$$

where $j_{z}^{0}(k, z)$ is the Hankel transform of the electric current density of the zeroth kind (see (19)). Accordingly, for $b_{0}(k)$ we have

$$
b_{0}(k)=b_{\phi}^{1}(k, 0)=\frac{j_{z}^{0}(k, 0)}{k} .
$$

We will assume that the layer at the lower boundary rests on a semi-infinite solid medium of the same conductivity (in reality, the thickness of this solid layer must be of the order of or greater than the thickness of the fluid layer). In this case, the magnetic field continuity condition must be met:

$$
\frac{\partial b_{z}^{0}}{\partial z}-k b_{z}^{0}=0, \quad \frac{\partial b_{\phi}^{1}}{\partial z}-k b_{\phi}^{1}=0 .
$$

System (22)-(29) is a system of linear differential equations with constant coefficients, which allows its solution to be sought in the form $f \sim \exp (-s z)$, where $s$ satisfies the tenth-order characteristic equation

$$
\begin{gathered}
\left(s^{2}-1\right)\left[\left(\left(s^{2}-k^{2}\right)^{2}-\mathrm{Ha}^{2} s^{2}\right)^{2}\right. \\
\left.+4 \operatorname{Re}^{2} s^{2}\left(s^{2}-k^{2}\right)\right]=0 .
\end{gathered}
$$

If the roots of the characteristic equation are known, then the solution of system (22)-(29), along with the boundary conditions (31)-(37), can be found exactly. However, both seeking for the roots of the characteristic equation (38) and the subsequent solution of system (22)-(29) are too cumbersome. Below, we provide an approximate solution that turns out to be quite sufficient for our purposes and allows the solution to be obtained in an analytical form (via integrals). 


\section{APPROXIMATE SOLUTION}

Let us neglect the term $2 \mathrm{Re} u_{r}^{1}$ in Eq. (23) (below, we will test the applicability of this approximation). The equations for the variables $u_{\phi}^{1}$ and $b_{\phi}^{1}$ are then separated from the complete system

$$
\begin{gathered}
-\mathrm{Ha}^{2} \frac{\partial b_{\phi}^{1}}{\partial z}=\left(\frac{\partial^{2} u_{\phi}^{1}}{\partial z^{2}}-k^{2} u_{\phi}^{1}\right), \\
-\frac{\partial u_{\phi}^{1}}{\partial z}=\left(\frac{\partial^{2} b_{\phi}^{1}}{\partial z^{2}}-k^{2} b_{\phi}^{1}\right) .
\end{gathered}
$$

The characteristic equation of this system has a simple form:

$$
\left(s^{2}-k^{2}\right)^{2}-\mathrm{Ha}^{2} s^{2}=0 .
$$

The roots of this equation are defined by the expression

$$
s^{2}=\frac{\mathrm{Ha}^{2}+2 k^{2} \pm \mathrm{Ha}\left(\mathrm{Ha}^{2}+4 k^{2}\right)^{1 / 2}}{2} .
$$

Hence the relation between the roots is

$$
s_{1}=-s_{2}, \quad s_{3}=-s_{4}=\frac{k^{2}}{s_{1}} .
$$

As will be seen below, the relation $\mathrm{Ha}^{2} \gg k^{2}$ usually holds. In this case, we will obtain the following approximate expressions for the roots:

$$
\begin{gathered}
s_{1}=-s_{2}=\mathrm{Ha}\left(1+\frac{k^{2}}{\mathrm{Ha}^{2}}\right), \\
s_{3}=-s_{4}=\frac{k^{2}}{s_{1}}=\frac{k^{2}}{\mathrm{Ha}} .
\end{gathered}
$$

The general solution of system (39), (40) is

$$
\begin{gathered}
b_{\phi}^{1}=C_{b \phi}^{(1)} e^{s_{1} z}+C_{b \phi}^{(2)} e^{s_{2} z} \\
+C_{b \phi}^{(3)} e^{s_{3} z}+C_{b \phi}^{(4)} e^{s_{4} z} \\
u_{\phi}^{1}=C_{u \phi}^{(1)} e^{s_{1} z}+C_{u \phi}^{(2)} e^{s_{2} z} \\
+C_{u \phi}^{(3)} e^{s_{3} z}+C_{u \phi}^{(4)} e^{s_{4} z}
\end{gathered}
$$

where the coefficients $C_{b \phi}^{(i)}$ and $C_{u \phi}^{(i)}(i=1,2,3,4)$ are not independent but are related by the relations that follow from the equations of system (39) and (40):

$$
\begin{gathered}
C_{u \phi}^{(i)}=-\frac{s_{i} \mathrm{Ha}}{s_{i}^{2}-k^{2}} C_{b \phi}^{(i)}, \\
C_{u \phi}^{(i)}=\frac{k^{2}-s_{i}^{2}}{s_{i}} C_{b \phi}^{(i)} .
\end{gathered}
$$

Obviously, when the characteristic equation (41) holds, relations (47) and (48) are equivalent and are brought to the form

$$
C_{u \phi}^{(i)}=-\mathrm{Ha}_{b \phi}^{(i)}
$$

at $i=1$ and 4 and

$$
C_{u \phi}^{(i)}=\mathrm{Ha}_{b \phi}^{(i)}
$$

at $i=2$ and 3 .

Using the boundary conditions (31)-(33) and (37) and the more stringent condition $\mathrm{Ha} \gg k^{2}$ (below, we will see that this condition also holds good), we will obtain the expressions

$$
\begin{gathered}
u_{\phi}^{1}=\mathrm{Ha}_{0}(k)\left\{\frac{k}{\mathrm{Ha}}[1+k(1+z)\right. \\
\left.-\frac{k}{\mathrm{Ha}}\right]-\frac{k^{2}}{\mathrm{Ha}^{2}}\left(1+\frac{k^{2}}{\mathrm{Ha}} z\right) e^{\mathrm{Ha} z} \\
-\frac{k}{\mathrm{Ha}}\left[1-\frac{k}{\mathrm{Ha}}(1+(1+z) k)\right. \\
\left.\left.-\frac{k^{2}}{\mathrm{Ha}^{2}}\left(2-k(1+k) z-\frac{k^{2} z^{2}}{2}\right)\right] e^{-\mathrm{Ha}(1+z)}\right\} \\
b_{\phi}^{1}=b_{0}(k)\left\{1-\frac{k^{2}}{\mathrm{Ha}^{2}}[1-k(1+k) z\right. \\
\left.-\frac{k^{2} z^{2}}{2}\right]+\frac{k^{2}}{\mathrm{Ha}^{2}}\left[1+\frac{k^{2}}{\mathrm{Ha}} z\right] e^{\mathrm{Ha} z} \\
-\frac{k}{\mathrm{Ha}}\left[1-\frac{k}{\mathrm{Ha}}(1+k(1+z))\right. \\
\left.\left.-\frac{k^{2}}{\mathrm{Ha}^{2}}\left(2-k(1+k) z-\frac{k^{2} z^{2}}{2}\right)\right] e^{-\mathrm{Ha}(1+z)}\right\} .
\end{gathered}
$$

Note that the factors at the exponentials are written out with a higher accuracy. This is needed when they are substituted into the boundary conditions, because large factors Ha appear when the exponentials are differentiated with respect to $z$. In addition, Eq. (52) allows the variation of the radial electric current density component with depth to be calculated (it is proportional to $\left.\partial b_{\phi}^{1} / \partial z\right)$.

We see that the azimuthal velocity (51) changes abruptly in a narrow (Hartmann) layer near the lower solid boundary but behaves smoothly near the upper free boundary. The azimuthal magnetic field has a smooth behavior at both boundaries.

From Eq. (51) for the Hankel transform of the azimuthal velocity on the surface to within $k / \mathrm{Ha}$ we have

$$
u_{\phi}^{1}(0)=b_{0}(k) k(1+k),
$$

where $b_{0}(k)$ is determined by the electric current density on the surface (36).

For our subsequent calculations, we need to have a specific expression for the Hankel transform of the 
TSYGAN et al.
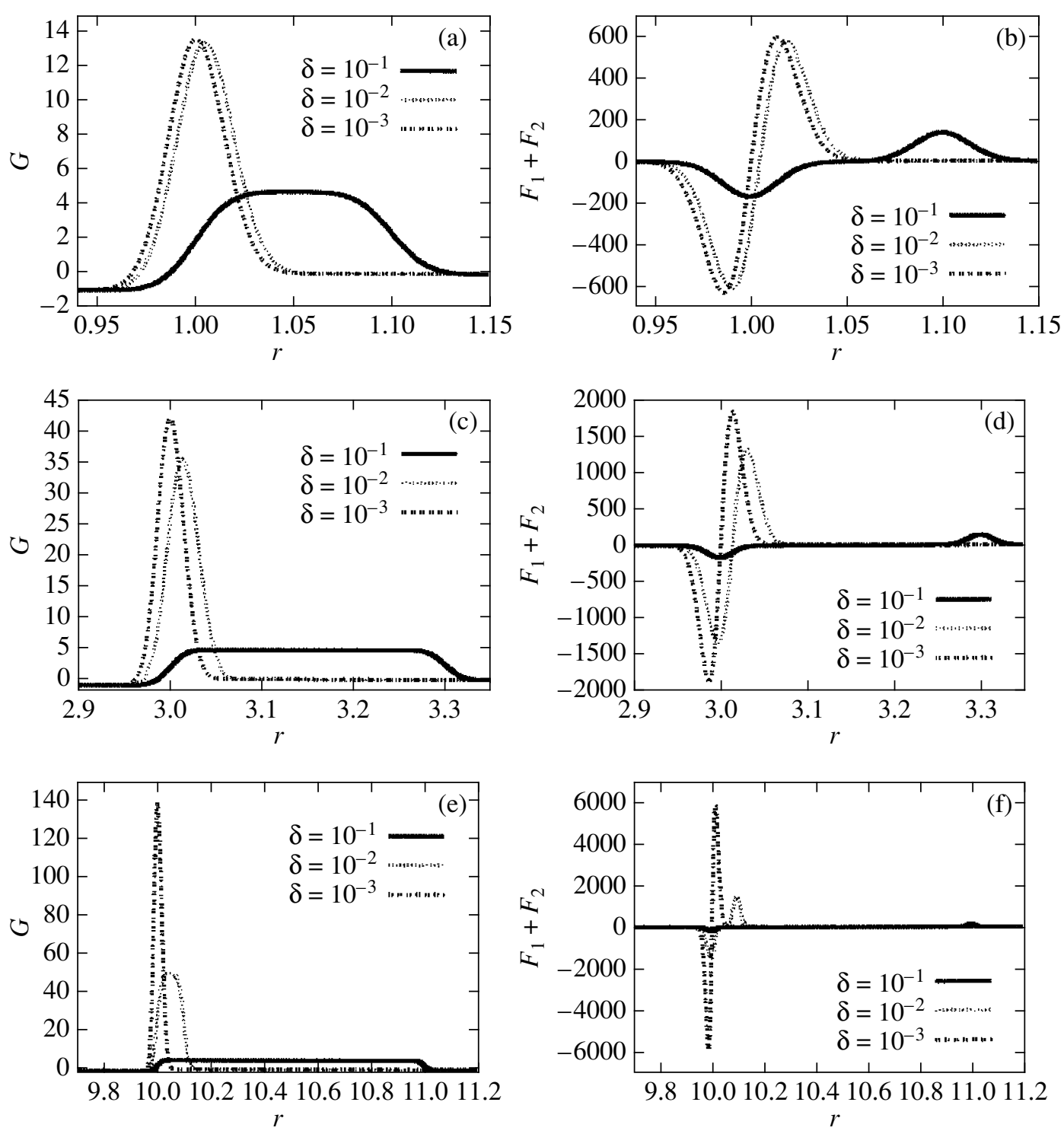

Fig. 1. The function $G$ defining the dimensionless current density (55) and the function $F_{1}+F_{2}$ defining the dimensionless azimuthal velocity (57) on the neutron star surface for $R_{1}=1(\mathrm{a}, \mathrm{b}), 3(\mathrm{c}, \mathrm{d}), 10(\mathrm{e}, \mathrm{f}), \beta=10^{-4}$. The values of $\delta$ are given in the figures.

electric current density on the surface. As this expression we will choose

$$
\begin{gathered}
j_{z}^{0}(k, 0)=j_{0} G^{0}(k)=j_{0} \frac{R_{1}^{2} R_{2}^{2}}{R_{2}^{2}-R_{1}^{2}} \\
\times \frac{1}{k}\left[\frac{1}{R_{2}} J_{1}\left(k R_{2}\right)-\frac{1}{R_{1}} J_{1}\left(k R_{1}\right)\right] e^{-\beta k^{2}},
\end{gathered}
$$

where $j_{0}$ is an arbitrary constant. Note that the current density can be calculated by using the inverse Hankel transform (18)

$$
j_{z}(r, 0)=j_{0} G(r)
$$

$$
G(r)=\int_{0}^{\infty} k G^{0}(k) J_{0}(k r) d k .
$$

In the limit $\beta \rightarrow 0$, we will obtain

$$
\begin{gathered}
G(r)=\frac{1}{R_{2}^{2}-R_{1}^{2}} \\
\times\left[R_{1}^{2} \theta\left(R_{2}-r\right)-R_{2}^{2} \theta\left(R_{1}-r\right)\right],
\end{gathered}
$$

where $\theta(r)$ is the Heaviside step function. In this limit, $G(r)=-1$ for $0 \leq r<R_{1}, G(r)=R_{1}^{2} /\left(R_{2}^{2}-\right.$ $\left.R_{1}^{2}\right)$ for $R_{1} \leq r<R_{2}$, and $G(r)=0$ for $r>R_{2}$. Thus, a uniform electric current with a density $-j_{0}$ is incident on the surface within the radius $R_{1}$ and a uniform electric current with such a density that the 

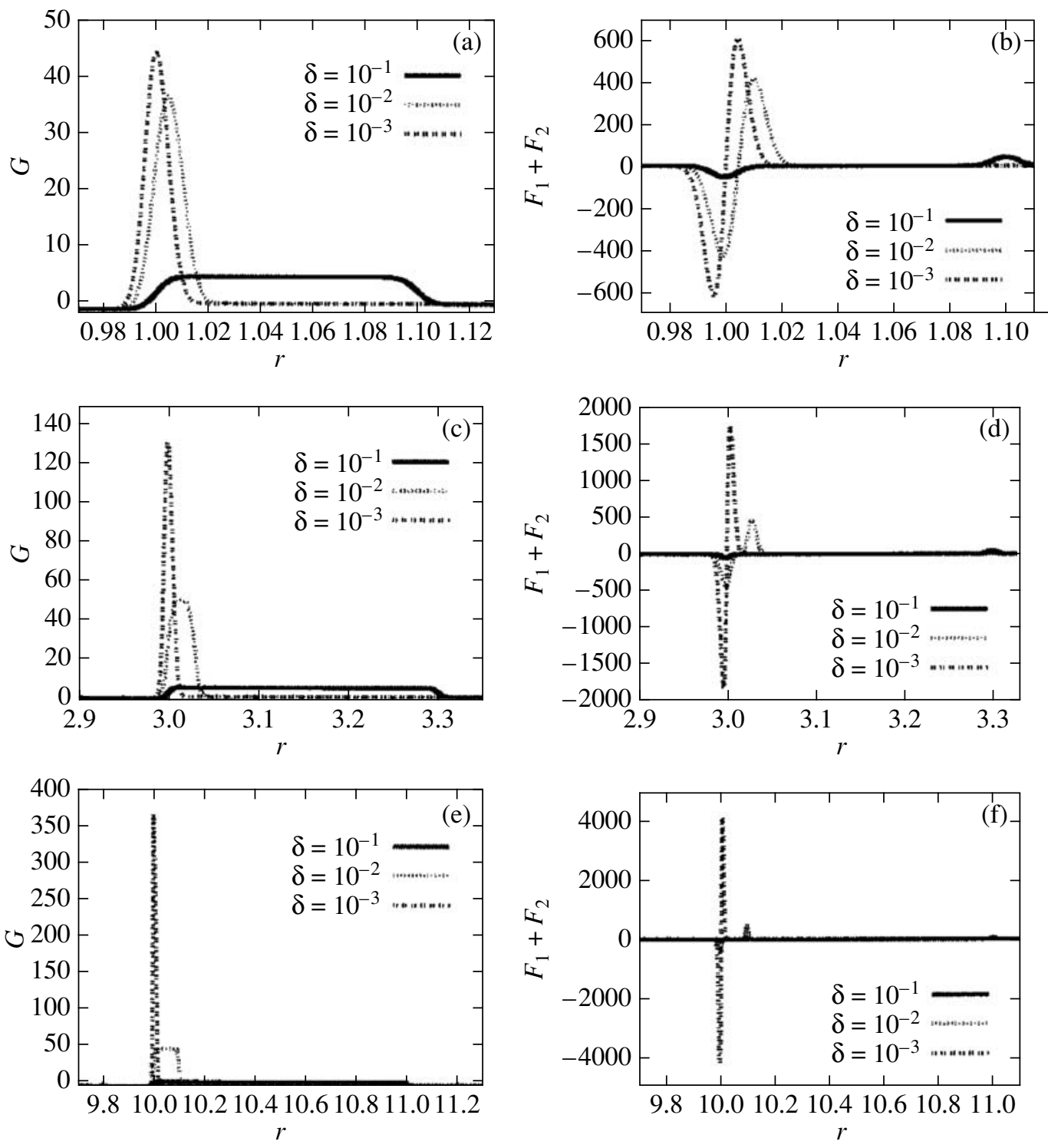

Fig. 2. Same as Fig. 1 for $\beta=10^{-5}$.

total current is zero flows out from the surface within the ring $R_{1}<r<R_{2}$. The parameter $\beta$ allows the function $j_{z}(r, 0)$ to be smoothed in such a way that the derivatives with respect to $r$ are everywhere finite. Note that the current density (54) qualitatively corresponds to the actual behavior of the electric current density in the region of the polar caps of neutron stars (see the Introduction).

Using (53), (54), and (36), we can calculate the azimuthal velocity and magnetic field components on the surface

$$
u_{\phi}(r, 0)=\int_{0}^{\infty} k^{2}(1+k) b_{0}(k) J_{1}(k r) d k
$$

$$
\begin{gathered}
=j_{0} \int_{0}^{\infty} k(1+k) G^{0}(k) J_{1}(k r) d k \\
=j_{0}\left[F_{1}(r)+F_{2}(r)\right], \\
F_{1}(r)=\int_{0}^{\infty} k G^{0}(k) J_{1}(k r) d k, \\
F_{2}(r)=\int_{0}^{\infty} k^{2} G^{0}(k) J_{1}(k r) d k, \\
b_{\phi}(r, 0)=\int_{0}^{\infty} k b_{0}(k) J_{1}(k r) d k
\end{gathered}
$$


TSYGAN et al.
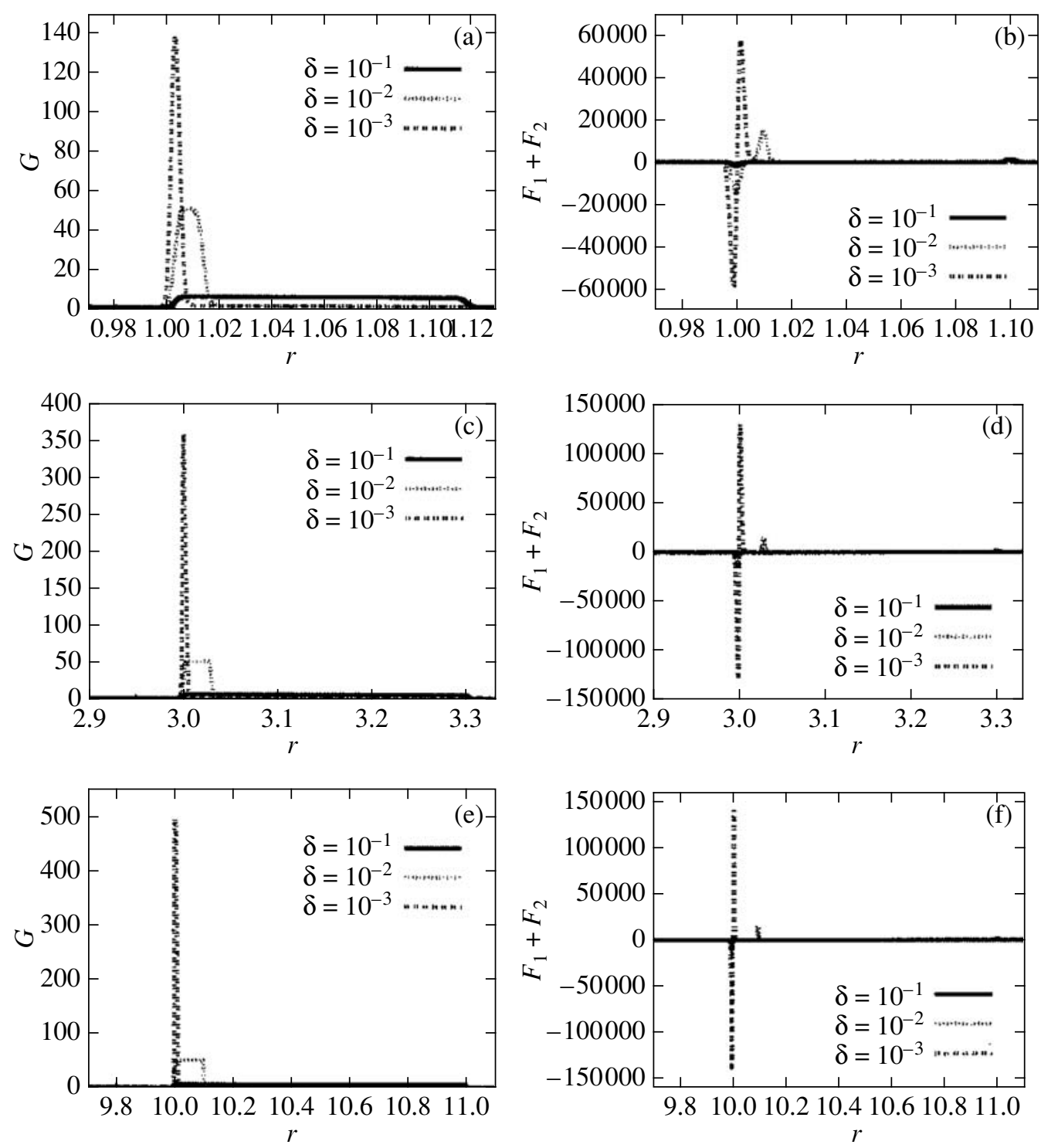

Fig. 3. Same as Fig. 1 for $\beta=10^{-6}$.

$$
=j_{0} \int_{0}^{\infty} G^{0}(k) J_{1}(k r) d r=j_{0} F_{0}(r) .
$$

Equations (57) and (59) depend on three dimensionless parameters, $R_{1}, \delta=\left(R_{2}-R_{1}\right) / R_{1}$, and $\beta$. Recall that the polar cap radius $R_{1}$ is normalized to the depth of the fluid layer $d$. In our numerical calculations, we used three different values of the radius, $R_{1}=1,3,10$. For the parameter $\delta$, it is reasonable to assume that it is much less than unity. We performed our calculations for $\delta=0.1,0.01,0.001$. The parameter $\beta$ defining the characteristic scale of the change in current density was chosen so that it was less than or of the order of $\delta$. Our calculations were performed for $\beta=10^{-4}, 10^{-5}, 10^{-6}$.
Figures 1-4 present plots of the functions $G$ and $F_{1}+F_{2}$ that define, respectively, the behavior of the dimensionless current density and the azimuthal velocity on the surface for the above values of the parameters. We see that the amplitude of the current density depends much more significantly on $\beta$ than on $\delta$ (at the same time, the amplitude increases as both $\beta$ and $\delta$ decrease). Note that saturation occurs at $R_{1}=10$ and the amplitude ceases to depend on $\beta$ (compare the plots for $\beta=10^{-5}$ and $10^{-6}$ ). Our calculations show that the function $F_{1}+F_{2}$ defining the azimuthal velocity on the surface (57) behaves similarly. At the points where the values of the functions differ significantly from zero, the inequality $\left|F_{2}(r)\right| \gg$ $\left|F_{1}(r)\right|$ holds. Accordingly, the azimuthal velocity presented in Figs. 1-3 is actually determined by the 

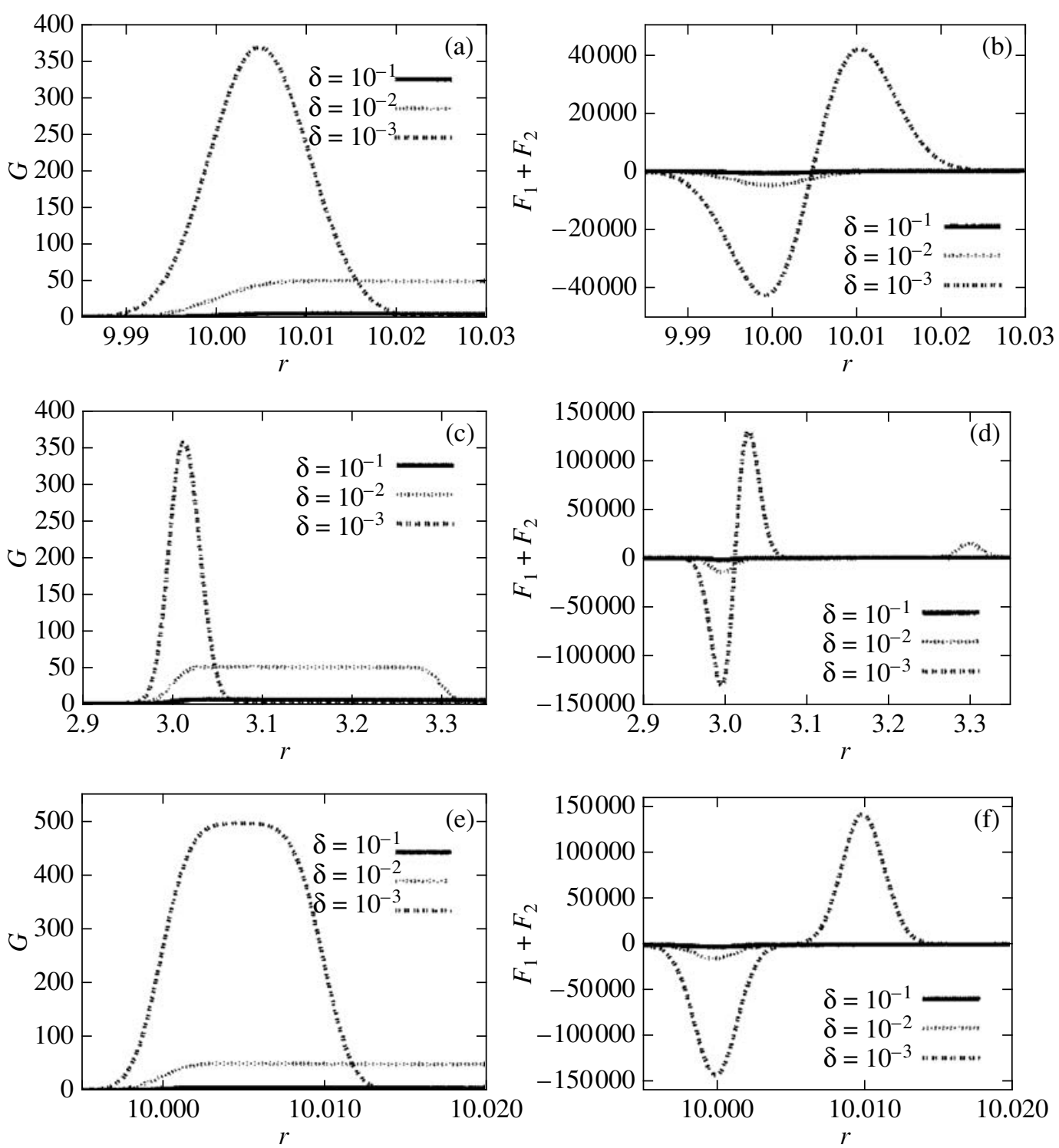

Fig. 4. Panel (a) shows the same as that in Fig. 2e; (b) the same as that in Fig. 2f; (c) the same as that in Fig. 3c; (d) the same as that in Fig. 3d; (e) the same as that in Fig. 3e, (f) the same as that in Fig. 3f but on a larger scale.

function $F_{2}(r)$. Note that the following relation holds:

$$
F_{2}(r)=-\frac{d G(r)}{d r},
$$

i.e., the azimuthal velocity on the surface is determined by the electric current density gradient with radius. Indeed, according to Figs. $1-4$, the azimuthal velocity changes significantly in narrow regions (the smaller the parameter $\beta$, the narrower they are) of change in the electric current density. Thus, two oppositely rotating narrow rings appear on the stellar surface.

Figure 5 presents the function $F_{0}$ defining the behavior of the azimuthal magnetic field on the surface for the electric current density distribution shown in
Figs. 1-4. We see that the behavior of the magnetic field depends weakly on the parameters. However, the amplitude of the azimuthal magnetic field depends significantly (directly proportional) on $R_{1}$. This behavior obviously follows from the fact that the azimuthal magnetic field is determined by the total current flowing within the ring of radius $r$. According to (54) and (55), the current density within the ring $R_{1}$ at small $\beta$ is virtually constant and the magnetic field grows linearly (in magnitude) with radius. Since the total current was assumed to be zero, the azimuthal magnetic field falls to zero on the scales determined by the parameter $\delta$ after the region of its linear growth.

To justify our approximation, let us estimate the velocity $u_{r}$. For this purpose, let us substitute 

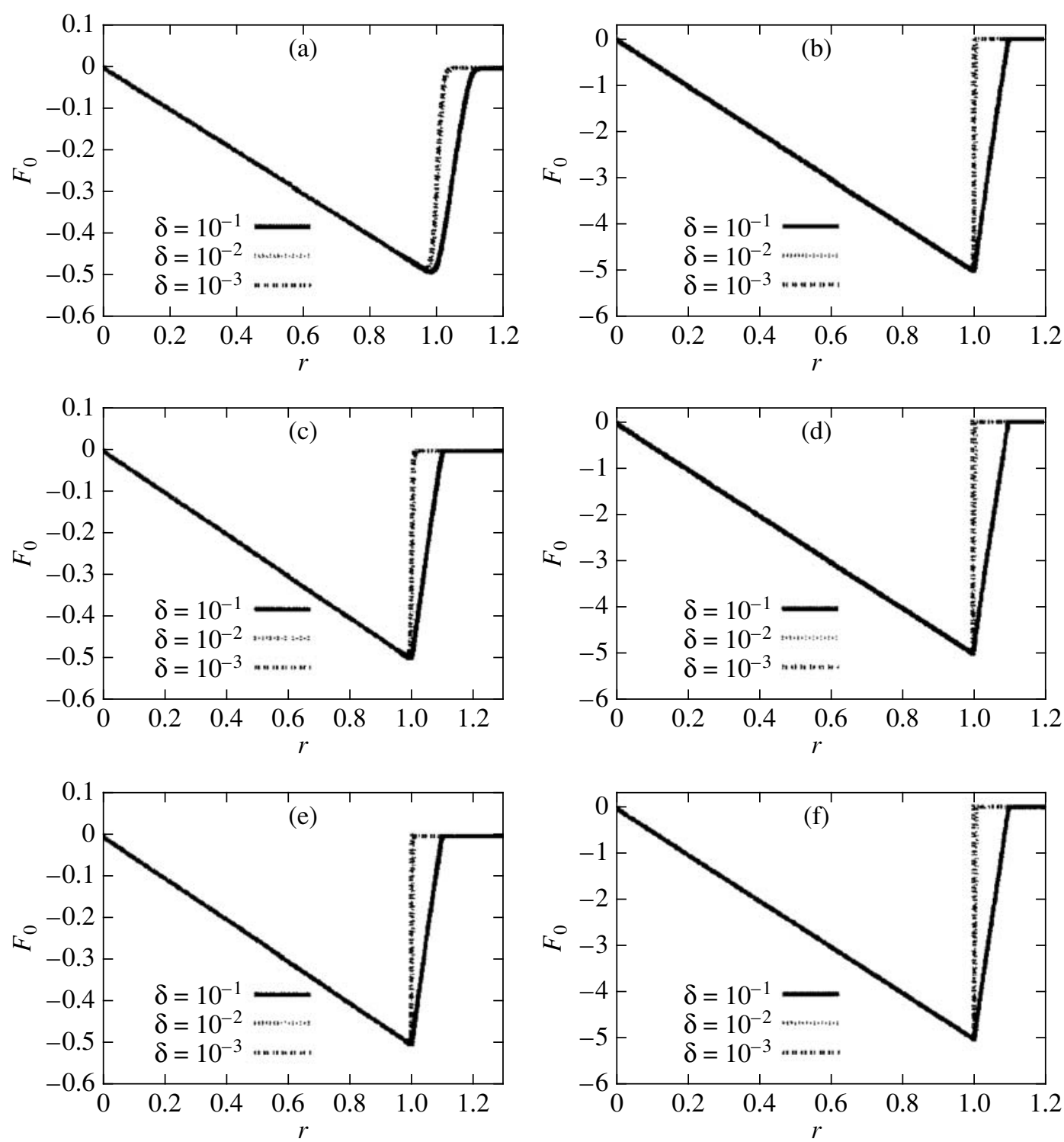

Fig. 5. The function $F_{0}(r)$ defining the azimuthal magnetic field on the surface (59) for $R_{1}=1$ (left) and 10 (right), $\beta=10^{-4}$ $(\mathrm{a}, \mathrm{b}), 10^{-5}(\mathrm{c}, \mathrm{d}), 10^{-6}(\mathrm{e}, \mathrm{f})$. The values of $\delta$ are given in the figures.

Eqs. (57) and (59) into the remaining equations of system (22)-(29) (except for Eqs. (23) and (26), which have already been used in deriving Eqs. (57) and (59)). Using the boundary conditions (31)-(33) and (37), we will obtain the following expression to within the terms $k^{2} / \mathrm{Ha}^{2}$ :

$$
\begin{gathered}
u_{r}^{1}(k, z)=\operatorname{Re} b_{0}(k) \frac{k}{\mathrm{Ha}}\left\{(1+2 z) \frac{k}{\mathrm{Ha}}\right. \\
-\left[1+z-\frac{k}{\mathrm{Ha}}-(1+z)^{2} \frac{k^{2}}{\mathrm{Ha}}\right] e^{-(z+1) \mathrm{Ha}} \\
\left.+\frac{k}{\mathrm{Ha}}\left[z-\frac{3}{\mathrm{Ha}}+\frac{k^{2} z^{2}}{\mathrm{Ha}}\right] e^{\mathrm{Ha} z}\right\} .
\end{gathered}
$$

Thus, the ratio of the Hankel transforms, along with the ratio of the radial and azimuthal velocities themselves, is $\mathrm{Re} / \mathrm{Ha}^{2}$ in order of magnitude. The corresponding term in Eq. (23) is smaller than the principal terms of the equation by a factor of $\mathrm{Re}^{2} / \mathrm{Ha}^{2}$, which is a very small number for typical parameters of the surface layers of neutron stars (see below) and, accordingly, our approximation is a good one. Note that $u_{z}^{1}$ can be estimated from the continuity equation and it will be of the order of $k u_{r}^{1}$.

For our numerical estimations, we will choose a density $\rho \sim 10^{4} \mathrm{~g} \mathrm{~cm}^{-3}$, a temperature $T \sim 10^{7} \mathrm{~K}$, a fluid layer depth $d \sim 3 \times 10^{3} \mathrm{~cm}$ (Haensel et al. 2007), a polar cap radius $R \sim 10^{4} \mathrm{~cm}$, an angular velocity of rotation $\Omega \sim 10 \mathrm{rad} \mathrm{s}^{-1}$, a neutron star radius $R_{\mathrm{ns}} \sim$ 
$10^{6} \mathrm{~cm}$, and a magnetic field $B \sim 10^{12} \mathrm{G}$ as typical values. In this case, the electric conductivity is $\sigma \sim$ $10^{18} \mathrm{~s}^{-1}$ (Potekhin 1999) and the kinematic viscosity is $\nu \sim 10^{-2} \mathrm{~cm}^{2} \mathrm{~s}^{-1}$ (Chugunov and Yakovlev 2005). Note that the influence of the magnetic field on the kinetic coefficients is disregarded. Accordingly, we have $\mathrm{Re} \sim 10^{10}$ and $\mathrm{Ha} \sim 10^{13}$. Thus, the parameter $\mathrm{Re}^{2} / \mathrm{Ha}^{2}$ is actually extremely small $\left(\sim 10^{-6}\right)$.

To estimate the actual differential rotation velocity in the polar cap of a neutron star, let us return to the dimensional quantities. Recall that the electric current density at the polar cap center is given by Eq. (1), where $\cos \chi=1$ for the aligned angular velocity and magnetic field vectors. Accordingly, $j_{0}$ from (54) takes the form

$$
j_{0}=\frac{2 \Omega d}{c}
$$

and for the dimensional azimuthal velocity on the surface $V(r)$ we have ( see (57))

$$
\begin{gathered}
V(r)=V_{0}\left[F_{1}(r)+F_{2}(r)\right] \\
=\frac{2 \Omega \eta}{c}\left[F_{1}(r)+F_{2}(r)\right] .
\end{gathered}
$$

For the above parameters, we will obtain $V_{0} \sim$ $10^{-7} \mathrm{~cm} \mathrm{~s}^{-1}$. Recall that the function $F_{1}+F_{2}$ is concentrated in narrow layers of change in the electric current density (Figs. 1-4), while its amplitude increases with decreasing $\delta$ and $\beta$. The maximum amplitude is $\sim 10^{5}$, corresponding to characteristic scales of the change in electric current density $\sim 3-$ $30 \mathrm{~cm}$. Thus, the maximum rotation velocity can reach $\sim 10^{-2} \mathrm{~cm} \mathrm{~s}^{-1}$, corresponding to a revolution time of $\sim 0.1-1 \mathrm{yr}$.

The typical values of the integration variable $k$ in Eqs. (51)-(53) and in integrals (58) and (59) are determined by the maximum value of two quantities, $1 / \sqrt{\beta}$ and $1 / \delta$, i.e., $k \sim 10^{3}$. Obviously, the condition $\mathrm{Ha} \gg k^{2}$ we use is met.

As has already been pointed out, the azimuthal velocity distribution on the surface in our model corresponds to two oppositely rotating rings. In this case, the inner ring always lags behind the main rotation.

\section{CONCLUSIONS}

We showed that differential rotation arises in the presence of an electric current in the region of the polar caps of magnetized neutron stars with a fluid surface. The properties of this rotation are determined by the magnitude and distribution of the electric current density over the surface. We considered the density distribution of a current consisting of two parts with opposite signs. In this case, the current of one sign flows in the region of open field lines, while the current of opposite sign flows in the region surrounding the region of open field lines. The total current through the surface is assumed to be zero. For such a structure of the current density, there exist two regions with an enhanced gradient (one in the interval of current sign reversal and the other in the outer region as the current falls to zero). Note that the direction of the current density in the region of open field lines corresponds to the fact that the force acting on the star brakes its rotation (the "-" sign in Eq. (1)). Given that the differential rotation velocity is determined mainly by the current density gradient (60), we will qualitatively obtain two oppositely rotating rings. Our estimates show that the typical azimuthal velocity on the surface is $10^{-2}-10^{-4} \mathrm{~cm} \mathrm{~s}^{-1}$, corresponding to a revolution time of $\sim 0.1-10 \mathrm{yr}$, while the typical radial and vertical velocities are much lower (approximately by 6 and 3 orders of magnitude, respectively).

\section{ACKNOWLEDGMENTS}

This work was supported in part by the Russian Foundation for Basic Research (project no. 1302-00112) and a grant from the President of Russia for Support of Leading Scientific Schools (NSh 294.2014.2).

\section{REFERENCES}

1. J. Arons and E. T. Scharlemann, Astrophys. J. 231, 854 (1979).

2. G. Bateman and A. Erdeii, Higher Transcendental Functions (McGraw-Hill, New York, 1953; Nauka, Moscow, 1966), Vol. 2, p. 85.

3. V. S. Beskin, A. V. Gurevich, and Ya. N. Istomin, Sov. Phys. JETP 58, 235 (1983).

4. V. S. Beskin, Axisymmetric Steady Flows in Astrophysics (Fizmatlit, Moscow, 2006) [in Russian].

5. V. S. Beskin, Y. N. Istomin, and A. A. Philippov, Phys. Usp. 53, 164 (2013).

6. A. I. Chugunov and D. G. Yakovlev, Astron. Rep. 49, 724 (2005).

7. P. Goldreich and W. H. Julian, Astrophys. J. 157, 869 (1969).

8. P. Haensel, A. Y. Potekhin, and D. G. Yakovlev, Neutron Stars (Springer, 2007), Vol. 1.

9. P. B. Jones, Astrophys. J. 209, 602 (1976).

10. E. M. Kantor and A. I. Tsygan, Astron. Rep. 47, 613 (2003).

11. L. D. Landau and E. M. Lifshitz, Course of Theoretical Physics, Vol. 8: Electrodynamics of Continuous Media (Nauka, Moscow, 1992; Pergamon, New York, 1984).

12. A. Y. Potekhin, Astron. Astrophys. 351, 787 (1999).

13. M. A. Ruderman and P. G. Sutherland, Astrophys. J. 196, 51 (1975).

14. E. T. Scharlemann, J. Arons, and W. M. Fawley, Astrophys. J. 222, 297 (1978).

15. P. A. Sturrock, Astrophys. J. 164, 529 (1971).

Translated by V. Astakhov 